\title{
Pleiotropic scaling and QTL data
}

\author{
Arising from: G. P. Wagner et al. Nature 452, 470-473 (2008)
}

Wagner et al. ${ }^{1}$ have recently introduced much-needed data to the debate on how complexity of the genotype-phenotype map affects the distribution of mutational effects. They used quantitative trait loci (QTLs) mapping analysis of 70 skeletal characters in mice ${ }^{2}$ and regressed the total QTL effect on the number of traits affected (level of pleiotropy). From their results they suggest that mutations with higher pleiotropy have a larger effect, on average, on each of the affected traits - a surprising finding that contradicts previous models ${ }^{3-7}$. We argue that the possibility of some QTL regions containing multiple mutations, which was not considered by the authors, introduces a bias that can explain the discrepancy between one of the previously suggested models and the new data.

Wagner et al. ${ }^{1}$ define the total QTL effect as

$$
T=\sqrt{\sum_{i=1}^{N} A_{i}^{2}}
$$

where $N$ measures pleiotropy (number of traits) and $A_{i}$ the normalized effect on the $i$ th trait. They compare an empirical regression of $T$ on $N$ with predictions from two models for pleiotropic scaling. The Euclidean superposition model ${ }^{3,4}$ assumes that the effect of a mutation on a given trait is independent of its effect on other traits. It predicts independence of $A_{i}$ and $N$ and therefore a square-root scaling of $T$ with $N$. The total effect model ${ }^{5-7}$ assumes that the total effect $T$ is independent of the number of affected traits $N$. As their data show a roughly linear dependence of $T$ on $N$, the authors reject both models. They conclude that not only the total effect, but, surprisingly, even the effect per trait increases with pleiotropy.

Although the authors address several problems in interpreting QTL data, there is one confounding factor not considered that could explain the most surprising part of their results. Crucially, whereas models address pleiotropy of single mutations, the data presented by Wagner et al. ${ }^{1}$ are on QTL pleiotropy. As QTLs correspond to relatively large genomic regions, they can contain more than a single mutation. The pleiotropic QTLs in this study correspond to regions of 3-50 centimorgans (ref. 2). Major QTLs can break down into the contributions of several genes ${ }^{8}$ and even multiple mutations in a single gene ${ }^{9}$, sometimes affecting different traits ${ }^{10}$. Multiple-mutation QTLs are likely in the present study ${ }^{1,2}$, for several reasons: first, the data are based on F2 mapping, which has limited power to resolve the effects of linked loci'. Second, as pleiotropy is the null-model ${ }^{2}$, there is a bias for grouping several QTLs into a single one. There is evidence that this may indeed be the case for all highly pleiotropic QTLs ${ }^{2}$. Third, for a single trait, a small number of QTLs is the nullmodel and indeed the study does not allow for more than two QTLs per trait on the same chromosome ${ }^{2}$. Last, fixation of different mutations at the same gene in the high- and low-selection lines seems plausible.

How would multiple mutations in some QTLs affect the results of the paper? The finding of low levels of pleiotropy is conservative. Indeed, pleiotropy of the single mutations can only be lower than pleiotropy of the QTLs. The effect on the scaling behaviour is more involved. Even if the effects of single mutations (and single-mutation QTLs) strictly follow the square-root scaling predicted by the superposition model, this is generally not true for QTLs with several mutations (Fig. 1). The predicted square-root dependency is reproduced only if the sets of traits affected by the different mutations in a QTL are non-overlapping. Overlapping sets of traits may produce an almost linear relationship of $N$ and $T$, and thus an apparent deviation from the superposition model (Fig. 1).

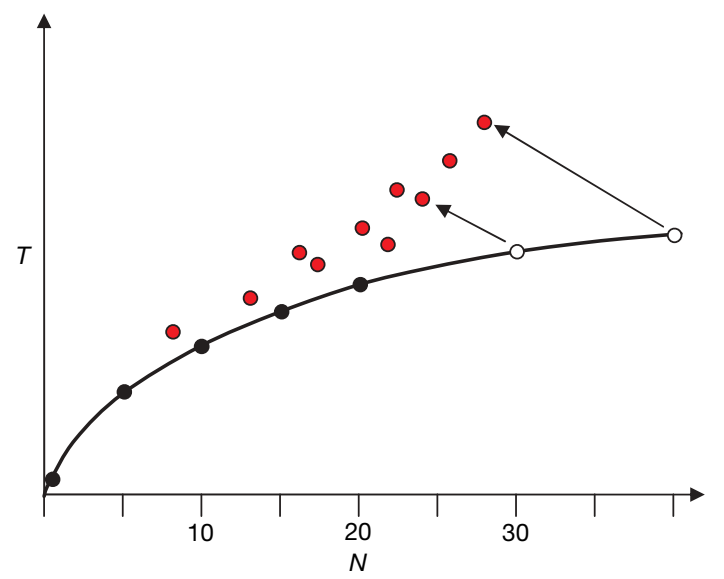

Figure 1 | Impact of multiple mutations on the total QTL effect, T. For all mutations, strict scaling according to the Euclidean superposition model is assumed. Black filled circles, single-mutation QTLs. Open circles, QTLs with two mutations that affect non-overlapping traits. Red filled circles, total effects of double-mutant QTL with overlapping trait ranges (see Methods). Arrows, shift due to the overlap.

Overlaps among sets of traits affected by different mutations in a single QTL are likely, particularly if each mutation affects many traits. Vice versa, QTLs with multiple mutations will (on average) affect a larger number of traits. We therefore expect the largest deviations from an overall square-root relation for data points with large $N$. This possibly explains the large deviations for the four QTLs with $N>25$ of Wagner et al. ${ }^{1}$. For example, a two-mutation QTL where both mutations affect $\sim 20$ traits could reproduce the observed results.

The $T-N$ regression is less affected by multiple-mutation QTLs if $T$ is measured in the linear Manhattan metric ${ }^{1}, T=\sum_{i}\left|A_{i}\right|$. Here, the superposition model predicts a linear regression. Overlaps in the trait sets of multiple-mutation QTLs lead to reduced $N$, but do not affect $T$. Indeed, the deviation of the data from a linear regression line appears to be small ${ }^{1}$.

Could multiple-mutation QTLs even reconcile the total effect model with the observed data? The answer is, in principle, yes, but only under extreme assumptions. In fact, we would have to explain the entire increase in $T$ with $N$ as solely due to ever-larger numbers of mutations in more pleiotropic QTLs.

Thus the data ${ }^{1}$ provide evidence against the total effect model, but not against the Euclidean superposition model. It therefore seems premature to accept a linear regression of the total mutational effect on the degree of pleiotropy or to ponder why mutations with higher pleiotropy should have a larger effect on each affected trait. Eventually, data from QTL fine-scale mapping will help resolve these issues by determining the number of mutations per QTL and the correlation among sets of traits affected by mutations in the same QTL region.

\section{METHODS}

Assume that all trait-specific mutational effects are equal to some constant $a$. The total effect $t$ of a mutation affecting $n$ traits is:

$$
t=a \sqrt{n}
$$

For single-mutation QTLs, $N=n$ and $T=t$, and data points follow the squareroot relation predicted by the superposition model (Fig. 1, black filled circles). The total effect $T$ of a multiple-mutation QTL $\left\{n_{1}, n_{2}, \ldots\right\}$ follows the same 
square-root law only if $N=\sum_{i} n_{i}$, that is, trait sets affected by the different mutations are non-overlapping (open circles in Fig. 1 for QTL $\{20,10\},\{20,20\}$ ). For overlapping trait sets, $N<\sum_{i} n_{i}$ and $T>a \sqrt{\sum_{i} n_{i}}$. For a two-mutation QTL with overlap $n_{12}, N=n_{1}+n_{2}-n_{12}$ and $T=a \sqrt{n_{1}+n_{2}+2 n_{12}}$. Red filled circles in Fig. 1 show $T$ and $N$ of all two-mutation QTLs with $n_{1} \geq n_{2} \in\{5,10,15,20\}$. The assumed overlap is $n_{12}=p\left(n_{1}\right) \cdot n_{2}$ with $p\left(n_{1}\right)=0.6$, $0.5,0.4,0.3$ for $n_{1}=20,15,10,5$, respectively.

Joachim Hermisson ${ }^{1} \&$ Alistair P. McGregor ${ }^{2}$

${ }^{1}$ Fakultät für Mathematik and Max F. Perutz Laboratories, University of Vienna, Nordbergstr. 15, 1090 Vienna, Austria.

e-mail: joachim.hermisson@univie.ac.at

${ }^{2}$ Institut für Populationsgenetik, Veterinärmedizinische Universität

Wien, Veterinärpl. 1, 1210 Vienna, Austria.

Received 29 May; accepted 2 September 2008.

1. Wagner, G. P. et al. Pleiotropic scaling of gene effects and the 'cost of complexity'. Nature 452, 470-473 (2008)
2. Kenney-Hunt, J. P. et al. Pleiotropic patterns of quantitative trait loci for 70 murine skeletal traits. Genetics 178, 2275-2288 (2008).

3. Turelli, M. Effects of pleiotropy on predictions concerning mutation-selection balance for polygenic traits. Genetics 111, 165-195 (1985).

4. Wagner, G. P. The influence of variation and developmental constraints on the rate of multivariate phenotypic evolution. J. Evol. Biol. 1, 45-66 (1988).

5. Orr, H. A. Adaptation and the cost of complexity. Evolution Int. J. Org. Evolution 54, 13-20 (2000).

6. Wingreen, N. S., Miller, J. \& Cox, E. C. Scaling of mutational effects in models of pleiotropy. Genetics 164, 1221-1228 (2003).

7. Martin, G. \& Lenormand, T. A general multivariate extension of Fisher's geometrical model and the distribution of mutational effects across species. Evolution Int. J. Org. Evolution 60, 893-907 (2006).

8. Orgogozo, V., Broman, K. W. \& Stern, D. L. High-resolution quantitative trait locus mapping reveals sign epistasis controlling ovariole number between two Drosophila species. Genetics 173, 197-205 (2006).

9. McGregor, A. P. et al. Morphological evolution through multiple cis-regulatory mutations at a single gene. Nature 448, 587-590 (2007).

10. Long, A. D., Lyman, R. F., Langley, C. H. \& Mackay, T. F. C. Two sites in the Delta gene region contribute to naturally occurring variation in bristle number in Drosophila melanogaster. Genetics 149, 999-1017 (2000).

doi:10.1038/nature07452

\section{Wagner et al. reply}

\section{Reply to: J. Hermisson \& A. P. McGregor Nature 456, doi:10.1038/nature07452 (2008)}

In our paper on pleiotropic scaling and the cost of complexity ${ }^{1}$, we presented evidence for three findings: first, most genes affect a small number of traits (the degree of pleiotropy is low); second, the total effect of a quantitative trait locus (QTL) increases with the degree of pleiotropy, refuting the constant total effect model $^{2,3}$; and third, the increase in total effect (defined as $T=\sum_{i} A_{i}^{2}$, where $A_{i}$ is the effect on character $i$, that is, half the difference between the genotypic values of the homozygous genotypes) seems to be stronger than predicted by the superposition model ${ }^{4,5}$ of pleiotropic effects. Hermisson and McGregor $^{6}$ point out that the last result could be due to multiple mutations being mapped to the same QTL, but only if these mutations affect overlapping sets of traits. We agree that this is a possibility that we could not address with the data at hand.

Hermisson and McGregor ${ }^{6}$ also point out that the total effect, if measured using a Manhattan metric, $t=\sum_{i}\left|A_{i}\right|$, is not affected by double mutations at a QTL, unlike the Euclidian metric we adopted. This is an important observation, as it can be used to estimate the overlap between the set of traits affected by the QTLs and their average pleiotropy. Applying this method to the four QTLs in our data set with the highest degrees of pleiotropy and which deviate

Table 1 | Analysis of the four highly pleiotropic QTLs

\begin{tabular}{lllll}
\hline$\hat{n}$ & $t$ & $\tilde{n}$ & $n_{12}$ & $\bar{n}$ \\
\hline 26 & 9.19 & 41 & 15 & 21 \\
28 & 7.35 & 33 & 5 & 17 \\
29 & 8.35 & 37 & 8 & 19 \\
30 & 8.69 & 39 & 9 & 20 \\
\hline
\end{tabular}

This analysis assumes that the effects of these QTLs are due to the combined effect of two mutations in each QTL. Here $\hat{n}$ is the number of affected traits, $t$ is the total effect, when measured as Manhattan distance, $\tilde{n}$ is the sum of the number of effects of both mutations based on the assumption that the effects per trait are invariant $(\tilde{n}=\hat{n} / 0.2236$, where the denominator is the regression coefficient of $t$ on $\hat{n}), n_{12}$ is the overlap of the trait sets affected by the two mutations $\left(n_{12}=\tilde{n}-\hat{n}\right)$, and $\bar{n}=\tilde{n} / 2$ is the average number of traits affected by the two mutations (average degree of pleiotropy). most strongly from the superposition model (see Supplementary Note 6 in ref. 1), we obtain the results shown in Table 1.

It follows from the final column of Table 1 that a double mutation model implies that the mutations at each double mutant-QTL have to have quite high degrees of pleiotropy (about 19 on average), which is unlikely given the distribution of pleiotropic effects in the rest of the data $(P=0.002)$. Hence the double mutation model, although plausible, is not closely compatible with our data. Resolution of the issue of whether we are faced with multiple mutations or a biological effect clearly requires data from more fine-grained QTL mapping experiments (J.M.C. et al., manuscript in preparation). But for now we agree with Hermisson and McGregor ${ }^{6}$ that the superposition model is a reasonable assumption about the pleiotropic scaling of mutation effects.

Günter P. Wagner ${ }^{1}$, Jane P. Kenney-Hunt ${ }^{2}$, Mihaela Pavlicev ${ }^{2}$, Joel R. Peck ${ }^{3}$, David Waxman ${ }^{3}$ \& James M. Cheverud ${ }^{2}$

${ }^{1}$ Department of Ecology and Evolutionary Biology, Yale University, New Haven, Connecticut 06520-8106, USA.

e-mail: gunter.wagner@yale.edu

${ }^{2}$ Department of Anatomy and Neurobiology, Washington University, St Louis, Missouri 63110, USA.

${ }^{3}$ Center for the Study of Evolution, School of Life Sciences, University of Sussex, Brighton BN1 9QG, UK.

1. Wagner, G. P. et al. Pleiotropic scaling of gene effects and the 'cost of complexity'. Nature 452, 470-472 (2008).

2. Orr, H. A. Adaptation and the cost of complexity. Evolution Int. J. Org. Evolution 54, 13-20 (2000).

3. Wingreen, N. S., Miller, J. \& Cox, E. C. Scaling of mutational effects in models of pleiotropy. Genetics 164, 1221-1228 (2003).

4. Turelli, M. Effects of pleiotropy on predictions concerning mutation-selection balance for polygenic traits. Genetics 111, 165-195 (1985).

5. Wagner, G.P. The influence of variation and of developmental constraints on the rate of multivariate phenotypic evolution. J. Evol. Biol. 1, 45-66 (1988).

6. Hermisson, J. \& McGregor, A. P. Pleiotropic scaling and QTL data. Nature 456, doi:10.1038/nature07452 (2008)

doi:10.1038/nature07453 